\title{
CAPÍTULO V \\ COSTOS OCULTOS, UNA HERRAMIENTA PARA \\ LA COMPETITIVIDAD DE LAS EMPRESAS CONSTRUCTORAS
}

\section{Eva Lozano Montero}

Doctora en Ciencias de la Administración, con línea de investigación en Finanzas. Profesora investigadora de la Universidad de Guanajuato. Coordinadora del Programa Emprendedor y Colaboradora en Ecosistema VIDA UG, monteroe@ugto.mx.

\section{Resumen}

En México y a nivel global, todas las empresas incurren en costos ocultos, los cuales se busca reducir; lo importante es detectarlos e implementar estrategias para su eliminación, por lo que el objetivo del presente trabajo de investigación es analizar que la implementación de la herramienta de costos ocultos en las empresas constructoras contribuya a la detección oportuna y control de los mismos, así como, a la permanencia y competitividad de las organizaciones, reflejándose en las utilidades y en el desarrollo organizacional. A través de un análisis exhaustivo de la literatura y considerando un estudio de caso referente a la industria de la construcción, en el presente estudio se evidencia que, al aplicar la herramienta de costos ocultos, se garantiza la competitividad en un mercado exigente y globalizado. Los resultados muestran los principales costos ocultos en el sector de la construcción y sugieren que, el desconocer los costos ocultos recurrentes en la operatividad de las constructoras, conlleva a decisiones que desencadenan costos ocultos adicionales y resultados desfavorables. El análisis de una adecuada gestión preventiva tanto en oficina central como en campo, así como la implementación de estrategias que contribuyan a evitar o reducir actividades innecesarias, repercute en el incremento del valor de la organización. Además, se determinó que, si se capacita al recurso humano y se detectan indicadores en el proceso de obra, contribuirán a la generación de valor de las constructoras.

Palabras clave: Competitividad, costos ocultos disfuncionamientos, sector construcción.

\section{HIDDEN COSTS, A TOOL FOR THE COMPETITIVENESS OF CONSTRUCTION COMPANIES}

\begin{abstract}
In Mexico and globally, all companies incur hidden costs, which are sought to reduce them, the important thing is to detect them and implement strategies for their elimination, so the objective of this research work is to analyze that the implementation of the tool hidden costs in the construction companies contributes to the timely detection and control of them, as well as, to the permanence and competitiveness of the organizations,
\end{abstract}


reflected in the profits and organizational development. Through an exhaustive analysis of the literature and considering a case study related to the construction industry, this study shows that by applying the hidden costs tool, competitiveness is guaranteed in a demanding and globalized market.They suggest that ignoring the recurrent hidden costs in the operation of construction companies leads to decisions that trigger additional hidden costs and unfavorable results. The analysis of adequate preventive management both in the central office and on the construction site, as well as the implementation of strategies that contribute to avoid or reduce unnecessary activities, has repercussions on the increase of organization's value. In addition, it was determined that, if human resources are trained and indicators are detected in the construction process, they will contribute to the generation of value by construction companies.

Keywords: Competitiveness, hidden costs, dysfunctions, construction sector.

\section{Capítulo resultado de un proyecto de investigación culminado.}

\section{Introducción}

En la economía nacional, la industria de la construcción es un sector de gran relevancia para el desarrollo nacional, proyectando un efecto multiplicador de empleos, la inversión en infraestructura impulsa el consumo del mercado interno, ya que al construir, no solamente genera empleos, sino que además activa el consumo de insumos para construir carreteras, aeropuertos, puertos, presas, drenajes, entre otros, como es el acero, madera, cemento y cobre, principalmente, facilitando así, el acceso al entorno globalizador y promoviendo la creación de negocios.No obstante, este sector es uno de los más vulnerables cuando en el país se presenta algún inconveniente económico, político o social, ya que perturba el desarrollo de la organización, exacerbando el enfrentamiento de problemas de solvencia, de liquidez, de absorción de costos y gastos fijos durante el lapso de tiempo que dejan de percibir ingresos. Lo anterior es preocupante para las empresas constructoras, principalmente para las MiPyMEs, que se ven obligadas a frenar su progreso y pierden oportunidades de desarrollo, además de que son las que presentan deficientes prácticas administrativas y operativas, incrementando la generación de costos ocultos.

Un costo oculto es aquel en el que incurre constantemente la empresa, y que no es necesario en la operatividad, pero es difícil de detectar, por lo que no se contabiliza y produce efectos negativos en los resultados contables, ya que tiene un costo. En el sector de la construcción, se enfrentan varios problemas, principalmente en la administración de obra, en donde se conjuntan conocimientos técnicos, de logística, administrativos, financieros, fiscales, entre otros., resultando actividades que no son necesarias, pero que se llevan a cabo de manera recurrente, convirtiéndose en costos ocultos. El presupuesto de obra es la clave para establecer el precio del inmueble, con el cual se participará en el concurso de obra y la eficiente determinación de los costos, coadyuva en el éxito de la organización. Mientras mejor control interno se tenga de los costos, tanto de los indirectos (oficina central y herramienta), como los de obra, se evitarán costos ocultos que se reflejarán en beneficios empresariales. 
De acuerdo con el Índice Global de Competitividad, la infraestructura es uno de los principales pilares de la competitividad, en donde México ocupa de manera general el lugar 51 y en infraestructura el lugar 62 de 137 naciones evaluadas. La infraestructura es uno de los 12 pilares básicos considerados en el indicador mencionado y en la edición 20172018, la calidad de la infraestructura carretera se encuentra en la posición 52, la calidad en infraestructura eléctrica se encuentra en el lugar 72 y la calidad de infraestructura en comunicaciones y la aeroportuaria en el lugar 67, siendo los indicadores más preocupantes en infraestructura. (CMIC, 2017). Sin embargo, en el ranking latinoamericano de infraestructura 2017-2018, México ocupa el cuarto lugar, considerado como uno de los que ha avanzado en comparación con años anteriores, aunque la inversión de 2016 a 2017 disminuyó en un 2.5\% respecto del Producto Interno Bruto (PIB), (CMIC, 2017). Es evidente que se deben escrutar mecanismos que impulsen la inversión en infraestructura, que contribuya a la calidad de vida de la población, al desarrollo económico y competitividad tanto a nivel regional y nacional.

La información existente sobre los costos ocultos en el sector de la construcción es limitada ya sea por la falta de estudios de investigación realizados al respecto, así como por la percepción desinteresada de las constructoras, principalmente de las micro y pequeñas empresas, las cuales tienden a desconocer su incidencia en el precio final de un inmueble, además de que las constructoras asignan un porcentaje predeterminado de costos indirectos por medio de una metodología establecida en el artículo 60 del Reglamento de la Ley de Obra Pública y Servicios Relacionados con la misma para el Estado y los Municipios de Guanajuato, lo que difiere de un estudio concienzudo de los costos en referencia.

De acuerdo al estudio de Lozano (2013), los problemas de financiamiento de las empresas no solamente se debe a la falta de opciones de crédito asequibles a las mismas, sino que también se debe a la ineficiente administración de los recursos, a la falta de personal especializado y a la inexistencia de agentes (teoría de agencias) que coadyuven al éxito de las mismas, provocando costos ocultos; que el gerente de la constructora no los percibe al momento de ser incurridos, ni los beneficios perdidos. La mayoría de las empresas incurren en costos ocultos sin percatarse de los mismos, se realizan diversas actividades por costumbre o por ser procesos habituales que no son analizados con la finalidad de mejorarlos o reducirlos y se siguen ejecutando de manera recurrente, contrarrestando la competitividad de las MiPyMEs constructoras principalmente.

Lo anterior, exacerba la pregunta de investigación de este estudio, que es: ¿La detección oportuna de los costos ocultos es una herramienta que coadyuva en la competitividad de las empresas constructoras? este cuestionamiento conlleva al objetivo implícito de analizar que la implementación de la herramienta de costos ocultos en las empresas constructoras contribuye a la detección oportuna y control de los mismos, así como, a la permanencia y competitividad de las organizaciones, reflejándose en las utilidades así como en el desarrollo organizacional.

Existen autores que confirman que los costos ocultos en que se incurre en el desarrollo de un proyecto de obra, se pueden controlar hasta tal punto que el precio final de un inmueble se optimice, lanzándolo al mercado con mejores oportunidades de éxito que las que pueda lograr la competencia (Navarrete \& Parra, 2012; Alarcón \& Campero, 1999; Botero \& Álvarez, 2003). 


\section{Fundamentación teórica}

Existe una gran variedad de costos ocultos, dependiendo de la actividad preponderante de cada sector y de la forma de trabajar de cada empresa; en este caso nos enfocaremos a la investigación de los costos ocultos en el sector de la construcción, siendo evidente que algunos costos ocultos están presentes en todos los sectores productivos.Para definir y entender los costos ocultos, primero se debe conceptualizar el término costo. Costo son los recursos sacrificados o perdidos para alcanzar un objetivo específico (Hongren \& Foster, 1992). Para García (2003), costo es "el valor monetario de los recursos que se entregan o prometen entregar a cambio de bienes o servicios que se adquieren”. Coincidiendo con la definición anterior, Ramírez (2008), define costo como "la suma de erogaciones en que incurre una persona física o moral para la adquisición de un bien o servicio, con la intención de que genere ingresos en el futuro".

Una vez conceptualizado lo que es costo, podemos definir los costos ocultos; que para Mejía (2008, p.5), "Los costos ocultos se encuentran presentes en el ciclo de producción, pero no son fácilmente cuantificables o asignables a las unidades producidas o vendidas". Los costos ocultos son aquellos que no se pueden detectar a simple vista porque se encuentran implícitos en el diario quehacer de la ejecución de cualquier proyecto y sólo se ven reflejados en el momento de evaluar los resultados o incluso después de la primera valoración, al desear realizar acciones para mejorar los procesos, (Navarrete \& Parra, 2012). Es por ello, que los costos ocultos no pueden reflejar en primera instancia la realidad de los beneficios esperados frente a los obtenidos. Se entiende por costos ocultos aquellos gastos que no son productivos o estrictamente necesarios para el funcionamiento de la empresa y que suelen pasar desapercibidos para los sistemas contables y la cuenta de resultados (Fernández, 2013). Guerrero (2012), define el costo oculto como un costo adicional que genera una transacción por no realizarse adecuadamente, el cual, generalmente no se conoce y es difícil de cuantificar.

En un análisis para identificar los costos ocultos y potencializar los ahorros, los factores más importantes a considerar son la frecuencia, el período y la cantidad. En este aspecto debemos incluir también el impacto de los costos ocultos en el entorno del proceso o sistema donde ocurre. Este problema realmente se presenta en todas las actividades y áreas de la construcción, e impactan fuertemente sobre la competitividad y rentabilidad. Dentro de un sistema de contabilidad o en el presupuesto de obra, si definimos los costosdesempeños ocultos como los datos que están registrados ya sea en el sistema contable o en el presupuesto de obra, los cuales se reflejan en cuentas analíticas y estados financieros; los costos-desempeños ocultos son los elementos de gastos y de productos no evidenciados por este sistema de información. (Zardet \& Krief, 2006).Para que un costo sea evidenciado y visible, debe cumplir por lo menos tres características: Debe ser identificado, o sea tener un nombre, una medida y tener un sistema de control continuo (Savall, 2013; Zardet \& Krief, 2006). Se considera que cualquier costo que no tenga estas características, entonces es un costo oculto, lo que puede observarse en la Figura No. 1.

La diferencia entre el funcionamiento esperado de una actividad (presupuesto de obra) y el funcionamiento real (ejecución de la obra), se le llama disfuncionamiento; en donde el factor principal que interviene en el mismo es el recurso humano (comportamiento) y es el más difícil de controlar. 


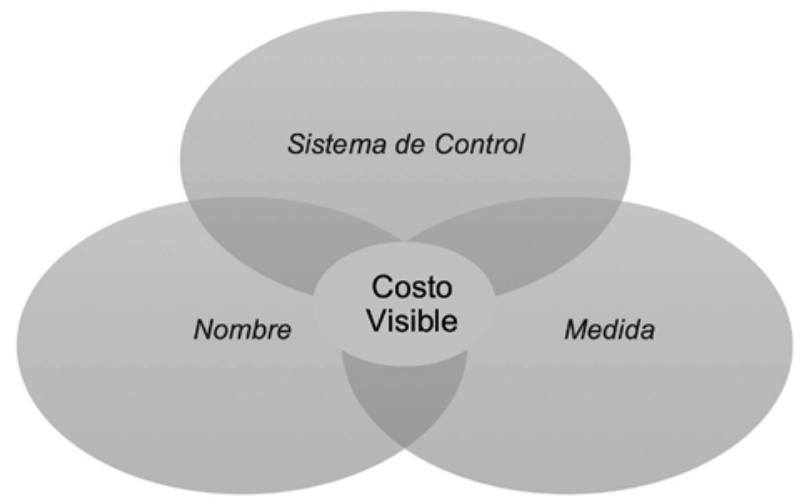

Figura No. 1. Características de un costo visible.

Fuente: Elaboración propia (2019), con información de Zardet y Krief (2006)

De acuerdo al resultado de trabajo del Instituto de Socio-Economía de las Empresas y de las Organizaciones (ISEOR), los costos ocultos representan pérdidas del 15\% al 30\% del costo de producción o del volumen de las ventas. Además, identifica seis principales disfuncionamientos que exacerban costos ocultos, los cuales se pueden identificar en indicadores del predominio social y del predominio económico. (Zardet \& Krief 2006), lo cual se observa en la (Figura No.2). Con ello, se pueden identificar y clasificar cualitativamente los costos ocultos y además cuantificar sus repercusiones económicas en la empresa. Los indicadores sociales y económicos permiten mejorar los procesos internos de las empresas, en donde es importante conocer el costo total del bien, sin embargo, también es necesario conocer otros elementos que ejercen un impacto negativo en la cadena de valor pero que se escapan de los métodos y herramientas de control de gestión tradicionales (Bampoky, 2012), estos otros elementos dependen de la actividad principal de la empresa, de la frecuencia en la que se incurre el costo y del monto del mismo.

Costos ocultos en constructoras. Todo empresario conoce cuáles son sus costos fijos por período, durante un lapso de tiempo determinado, pero los costos ocultos no se identifican fácilmente, aunque están presentes en la organización. Navarrete \& Parra (2012), recomiendan algunos aspectos a considerar en la elaboración de presupuestos, evitando así incrementos de costos reales que podrían provocar costos ocultos, como son los cambios en los costos unitarios, que son resultantes del aumento de los materiales; así como mezclar costos ocultos de manera inconsciente que resultan en un elevado presupuesto del proyecto, disminuyendo la competitividad de la empresa. Además, se debe considerar las distancias entre la oficina central y el lugar de trabajo, así como el riesgo que implica este tipo de oficios, los cuales son considerados de alto riesgo, siendo imperativo incorporar las erogaciones adicionales a la mano de obra, como el seguro social, equipo de protección y otros. 
Disfuncionamientos

- Condiciones del Trabajo

- Organización del Trabajo

- Gestión del tiempo

\section{Costos Ocultos}

- Ausentismo

- Accidentes de Trabajo

- Rotación de Personal
Indicadores de Predominio Social

\section{Disfuncionamientos}

- Comunicación, coordinación

- Formación integrada

- Implementación estratégica

\section{Costos Ocultos}

- No calidad del producto

- Baja productividad

- Duplicidad de actividades
Indicadores de Predominio Económico

Figura No. 2. Disfuncionamientos y Costos Ocultos.

Fuente: Elaboración propia (2019), con información obtenida de Savall \& Zardet (2006)

Los costos ocultos se detectan cuando se miden las áreas estratégicas con indicadores, pero si la empresa carece de indicadores, se dificulta la identificación de disfuncionamientos. Martínez (2015) nos proporciona una clasificación de costos ocultos y provee la siguiente información: costos por obsolescencia de equipos, costos de reposición, costo por mala comunicación, costos por inflación, costos financieros, costos por operación logística y distribución, costos por mala planeación de la producción y costos por mala calidad. Aunado a los anteriores, los costos ocultos más comunes en el proceso de obra son los siguientes: costo oculto de logística de tiempos muertos de entrega, costos ocultos de cumplimiento de normatividad, costos ocultos por manejo de inventario, costos por el flujo de caja, costos ocultos por accidentes de obra, costos ocultos por rotación del personal. (Pallarés, 2000; Navarrete y Parra, 2012)

Los costos ocultos no los absorbe el producto (la obra), estos son pagados por la empresa, debido a que, al no ser identificados, ni contabilizados, en el proceso de producción no son considerados.

\section{Caso práctico}

Se presenta un caso real en una empresa constructora de Villahermosa, Tabasco, que por cuestiones de confiabilidad de la información, se omite el nombre de la misma. Con el fin de detectar, identificar y analizar los costos ocultos que se presentaron, se dan a conocer las principales actividades de la obra en específico, demostrando una comparativa de los costos 
presupuestados (precios unitarios en obra), con los costos reales o ejecutados, así como los avances de obra. Asimismo, se muestran las pérdidas obtenidas y las repercusiones, que se reflejan en una pérdida de confianza y competitividad.

Antecedentes. La constructora se dedica a la elaboración de proyectos de obra, a la construcción de carreteras y obra civil, teniendo contratos con dependencias de gobierno y con la iniciativa privada, existe un período en el que firma varios contratos con ambos sectores, por lo que se ve obligada a incrementar su plantilla de personal, así como a la adquisición y arrendamiento de maquinaria. Realiza contratos de residentes de obra que son el pilar del control de la misma, teniendo la responsabilidad de vigilar y controlar el desarrollo de los trabajos contratados por ejecutar, cuidando la calidad especificada, además de los costos ejecutados y llevar una bitácora, entre otras funciones.

Desarrollo. En la obra se iniciaban los trabajos a las 7:00 am y se terminaban a las 18:00, el residente de obra llegaba a las 9:30 am, proporcionaba algunas indicaciones y se retiraba; está situación ocasionó una carencia del liderazgo en el trabajo, provocando tiempos ociosos, carencia de autoridad y costos ocultos de material, aunque principalmente de mano de obra, además de un retraso en la ejecución de obra. La obra tenía programado un tiempo de 6 meses, la cual solamente se ejecutó en 9 semanas, debido a que los avances no eran contundentes, por lo que se decide rescindir el contrato. Esta decisión no solamente afecta a la empresa de manera económica, ya que además trasciende en confianza y competitividad ante futuros clientes, tanto de obra pública como de obra privada. Las principales debilidades detectadas en la obra mencionada fueron, la ausencia del residente de obra y por consecuencia el ausentismo de los trabajadores, maquinaria con tiempo ocioso, trabajos con deficiente calidad, robo hormiga de materiales, herramientas y combustibles, además de dos accidentes de trabajo debido a que los trabajadores llegaban en estado de ebriedad a trabajar. Por lo anterior, la empresa constructora presenta un problema que está considerado dentro de los indicadores sociales de costos ocultos, denominado, ausentismo; además de gastos en gasolina, sin obtener los resultados esperados.

En este estudio de caso se muestran los disfuncionamientos de combustible y de mano de obra, los cuales se convierten en costos ocultos que se reflejan en una disminución en los resultados financieros, en el prestigio y competitividad de la constructora. En las figuras No. 3 y 4, se muestran los costos ocultos de combustible y mano de obra, realizando una comparativa entre costos presupuestados y costos reales. Durante las nueve semanas que se llevó a cabo la construcción, se observa que existen diferencias sustanciales de combustible y de mano de obra de $\$ 61,219.00$ y $\$ 28,082.00$ respectivamente. Gráficamente se puede apreciar los disfuncionamientos por ausentismo, falta de responsabilidad, desperdicios y robo de combustible. 


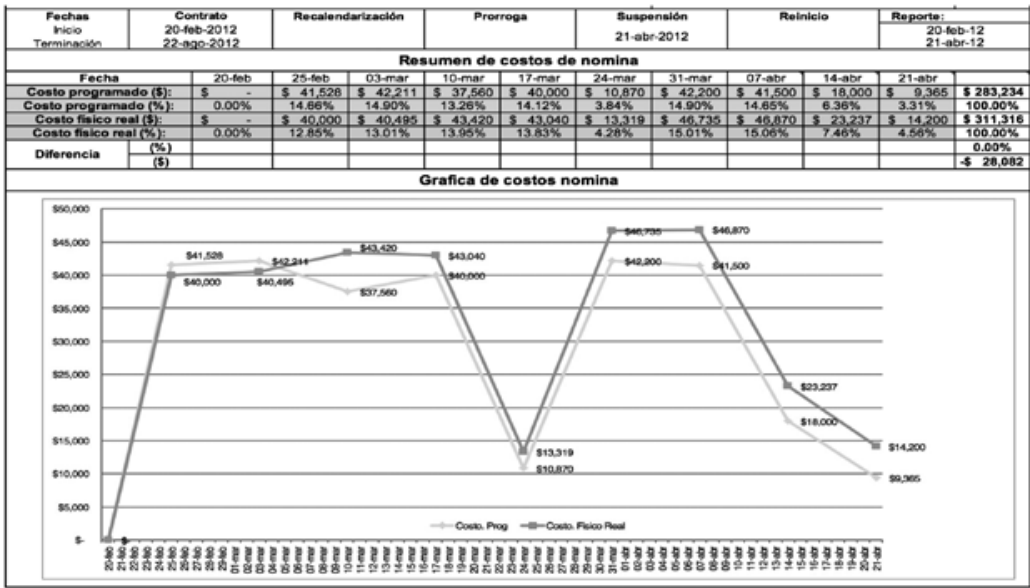

Figura 3. Disfuncionamiento de Costo de Obra de Combustible.

Fuente: Elaboración propia (2019), con información de la obra del estudio de caso.

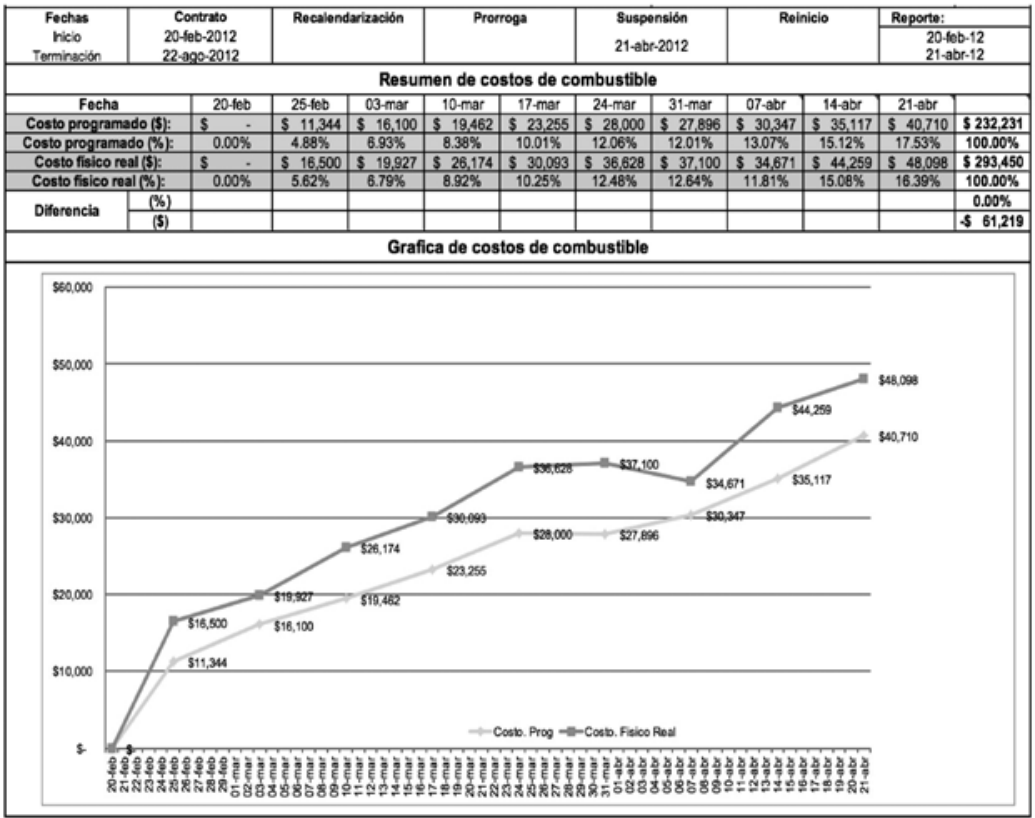

Figura 4. Disfuncionamiento de Costo de Obra de Mano de Obra.

Fuente: Elaboración propia (2019), con información de la obra del estudio de caso. 
Asimismo, para poder ejecutar los trabajos de obra, se realizaron contratos de renta de maquinaria, la cual no se utilizó para los fines que fueron destinados, obteniendo horas-máquina ociosa que se refleja en flujos negativos, ya que de acuerdo a la programación en Project, de donde se tenía programado a la novena semana, una ejecución de recursos de $\$ 580,273.00$, las erogaciones reales fueron de $\$ 697,450.00$, además de que no correspondían al avance de obra estimado, arrojando un saldo negativo de $\$ 117,177.00$ desde el punto de vista económico, aunque si se compara con el avance de obra, las pérdidas son mayores. Al percatarse del retraso, se rentaron más máquinas para alcanzar el avance programado, aumentando los costos. De acuerdo a los registros, el avance de ejecución de obra durante las nueve semanas de trabajo realizado, antes de ser rescindido el contrato, se programó de $46.67 \%$, mientras que lo ejecutado real resultó de $25.80 \%$. Los costos ocultos totales considerando los indicadores más relevantes -Combustible, Mano de Obra y Renta de Maquinaria- ascendieron a \$206,477.89

\section{Metodología}

Mediante una revisión exhaustiva de la literatura, se analiza la importancia de la detección oportuna, reducción y control de los costos ocultos en el sector de la construcción, como una herramienta en la competitividad de las empresas, en donde se describe cómo se han detectado los costos ocultos en las constructoras y los beneficios obtenidos al participar en las convocatorias y en la ejecución de obra. Asimismo, se incluye un caso práctico en donde se puede observar que, si la empresa no realiza actividades de acuerdo al presupuesto de obra, o si las realiza con menor eficiencia, los costos incurridos se elevarán debido a que se están incurriendo en costos ocultos. En la revisión de la literatura se muestran los antecedentes y algunas consideraciones conceptuales esenciales en el análisis de costos ocultos en general, y en específico en la industria de la construcción. En el caso práctico, se muestra que la no detección oportuna de costos ocultos, repercuten en la permanencia y competitividad de la constructora.

\section{Resultados}

Los resultados de este trabajo resaltan el importante papel del sector de la construcción en la economía del país, gracias a la creación de empleos y con el efecto multiplicador en las actividades productoras y de servicio, sin embargo; por sus características depende de muchos factores externos que hacen que el sector presente ciclos de expansión y contracción. Las empresas constructoras con el fin de mantenerse en el mercado y ganar los concursos de obra, realizan una competencia, muchas veces desleal, reduciendo considerablemente los presupuestos de los proyectos. Esto no solamente afecta de manera económica a la firma, sacrificando utilidades, sino que además afecta la calidad de la obra, que repercute en quejas, lo que exacerba costos ocultos.

La herramienta de costos ocultos, se constituye para que las empresas determinen en sus proyectos qué actividades son productivas, qué actividades se deben reducir de manera paulatina y qué trabajos se pueden eliminar. Esto les permite identificar las tareas que no contribuyen en el proceso y se pueden eliminar del ciclo de producción, reduciendo costos y mejorando la productividad y por lo tanto su competitividad. Los resultados obtenidos ponen de manifiesto que los principales costos ocultos detectados en el sector de la construcción son 
los siguientes: a) Costos por ausentismo; b) Costos de logística; c) Costos de mala calidad de obra; d) Costos por accidente de obra; d) Costos por incumplimiento de la normatividad y e) Costos por robo de herramienta y material. La investigación propone que una manera de detectar costos ocultos en las constructoras es comparar lo presupuestado con lo ejercido a cierto nivel de avance de obra, siendo una ventaja en las constructoras, ya que realizan una planeación y se pueden medir los avances e identificar indicadores, realizando ajustes necesarios, facilitando la detección de costos que están fuera de lo presupuestado.

\section{Discusión}

La prevención, control y cuantificación de los costos ocultos coadyuva en la eficiente ejecución de recursos de las empresas constructoras y facilita su ajuste en el proceso de obra, reflejándose en beneficios y posicionándose en el mercado. El control de costos ocultos se relaciona ampliamente con el recurso humano, siendo el que puede controlar y evitar una gran porción de ellos; a partir de los trabajos en los seis campos de las disfunciones en los costos y su identificación, (Savall \& Zardet, 2006), (Martínez, 2015), se busca reducir el impacto en las organizaciones, lo que redundará en la mejora de sus resultados financieros, sin que esto lleve al deterioro de su desempeño social, al contrario, este método, de manera indirecta motiva a las organizaciones a ser más responsables socialmente, ya que se busca optimizar los recursos naturales y a una mejor relación con sus empleados, por tanto, el control de gestión se pone en el centro de la relación entre el desarrollo económico de las empresas y la responsabilidad social, generando capital humano (Meyssonnier \& Rasolofo, 2008), (Cappelletti \& Levieux, 2010).

Se proponen los indicadores como una forma de detectar costos ocultos, aunque para el sector de la construcción, los elementos del presupuesto de obra pueden ser considerados como tal, ya que utilizan un software especializado en análisis de costos, además de que se auxilian de costos unitarios que son publicados y están actualizados; lo anterior, no descarta la posibilidad de que las empresas constructoras implementaran sus indicadores que apoyen en la detección de disfuncionamientos. Estos indicadores deben ser considerados en la toma de decisiones que modifica de forma importante el direccionamiento de costos y el organizacional. Asimismo; cuando se propone una estrategia, se debe verificar en qué punto ésta impacta los costos ocultos y determinar su viabilidad. Al implementar la metodología de detección de costos ocultos, se esperan resultados positivos que no se reflejan inmediatamente, se debe tener paciencia, ya que solo en el mediano y largo plazo se reflejan los beneficios de su implementación, además de ser necesaria una gestión social, en el que observen los empleados un ambiente propicio para laborar, siendo el recurso humano el que genere soluciones, de acuerdo a su experiencia, ya que son los que interactúan en los diferentes procesos y conocen las áreas de oportunidad por superar.

\section{Conclusiones}

Generalmente los costos directos que son identificables y cuantificables con la manufactura de un bien o servicio, es muy claro su control, pero no es tan evidente con los costos indirectos y los gastos de operación, que para la industria de la construcción son los 
costos de oficina central o los indirectos; aunque sí se consideran para conformar el costo total de obra, creando las empresas bases de asignación o desarrollan fórmulas para calcular horas máquina, horas hombre, entre otras.

La relación con la producción de un bien se dificulta con algunos costos como los financieros o los intangibles originados en marcas, patentes o desarrollo de conocimientos, los cuales están presentes y se deben considerar, ocasionando que no se asignen a la obra y pareciera que son costos ocultos. Los costos ocultos se encuentran presentes, pero no son asignables a las unidades producidas; en este caso a las obras ejecutadas, debido a que en varias ocasiones son incurridos sin detectarse, por lo que no son absorbidos por el precio estipulado del bien, pero sí repercuten en las ganancias.

La importancia de identificar indicadores en la metodología de detección de costos ocultos, radica en que se implementan estrategias de erradicación de costos ocultos, facilitando que los participantes se acoplen fácilmente al cambio, contribuyendo así, a la competitividad de la organización. Cada indicador que carece de la generación de un beneficio, es un costo oculto que representa pérdidas y una fuente de recursos utilizados de manera ineficiente.

Para poder implementar la herramienta de identificación, control y reducción de costos ocultos, se requiere de un cambio cultural en todos los niveles de las empresas, que permita establecer indicadores y aplicar nuevas técnicas de planificación y control del proceso productivo, en el que el centro de la aplicación de la herramienta son el recurso humano. Para ello, es necesario capacitar al personal encargado de la planeación, ejecución y control de los proyectos en nuevas estrategias de gestión, convirtiéndose en facilitadores en la aplicación de los nuevos conceptos. El control de avance de obra y reducción de costos ocultos radica en el supervisor de obra, que como se observó en el estudio de caso es el que revisa lo presupuestado con lo ejercido, además de que la detección oportuna de costos ocultos es de gran utilidad, ya que obliga a la organización a la implementación de herramientas para su control o eliminación de los mismos.

Esta investigación puede ser la motivación para la indagación de otras herramientas que apoyen en el control de los costos ocultos, principalmente para la industria de la construcción, lo que contribuirá a la competitividad de las empresas constructoras.

\section{Referencias bibliográficas}

Alarcón, L. \& Campero M. (1999). Administración de proyectos civiles. Santiago de Chile. Ediciones Universidad Católica de Chile. p.322.

Bampoky, B. (2012). Les dysfonctionnements révélateurs de coûts cachés dans les entreprises sénégalaises: quelles perspectives pour la maximisation de la valeur? Revue Congolaise de Gestion, pp.127-166. Disponible en: www.cairn. info/revue-revue-congolaise-de-gestion2012-1-page-127.htm

Botero, L. y Álvarez, M. (2003). Identificación de Pérdidas en el Proceso Productivo de la Construcción. Revista Universidad EAFIT no.130. 
Cámara Mexicana de la Industria de la Construcción, CMIC. Competitividad en Infraestructura : 2017-2018-CMIC. Consultado el 8 de febrero de 2018 en www. cmic.org.mx/cmic/ceesco/.../PRESENTACIÓN\%20COMPETITIVIDAD\%20. pdf

Cappelletti, L. \& Levieux, P. (2008). Le contrôle de gestion socio-économique : Convertir les coûts cachés en performance durable. Tomado de Nicolas Berland \& FrançoisXavier Simon. Le contrôle de gestion en mouvement: Etat de l'art et meilleures pratiques - Regards croisés de professeurs et praticiens, 63-78. Paris: Editions d'Organisation.

Fernández, N. (2013). Consentio Startegic Partners. Consultoría News. Costos Ocultos.

García, J. (2003). Contabilidad de Costos. McGraw Hill. Segunda edición. Mexico, D.F. p. 10

Guerrero, J. (2012). Costos Ocultos, Gestión Logística. Consultado el 2 de julio de 2017 en https://prezi.com/dgebnocrosoz/costos-ocultos/

Hongren, C., Foster, G, (1992). Contabilidad de costos, un enfoque gerencial. Prentice Hall, Quinta edición. México, D.F. p.23

Lozano, E. (2013). Percepción de las MiPyMEs constructoras acerca de los apalancamientos financieros: El Estado de Guanajuato como estudio de caso. XVIII Congreso Internacional de Contaduría, Administración e Informática; Capítulo 11 pp.127 UNAM, México, D. F., Octubre. ISBN 978-607-02-4620-3

Martínez, O. (2015). Costos Ocultos. Instituto Tecnológico de Buenos Aires. (ITBA)

Mejía, C. (2008), Documentos planning. Consultado el 8 de agosto de 2017, de www. planning.com.co/bd/archivos/Junio2008.pdf

Meyssonnier, F. \& Rasolofo-Distler, F. (2008). Le contrôle de gestion entre responsabilité globale et performance économique: le cas d'une entreprise sociale pour l'habitat. Management Control between Global Responsability and Economic Performance. The Case of a Social Firm for Housing. Comptabilité, Contrôle, Audit, 14 (2) 107-124. DOI: 10.3917/cca.142.0107

Navarrete, J. \& Parra, G. (2012). Costos Ocultos en el Proceso de Construcción en la Empresa Constructora, Amarilo, SAS. Universidad Militar de Nueva Granada. Facultad de Ciencias Económicas Especialización en Control Interno. Bogotá.

Pallarés, M. (2000). Costos ocultos en la producción inmobiliaria. Revista de Urbanismo. Edición del Departamento de Urbanismo de la F.A.U. de la Universidad de Chile, Marcoleta.

Ramírez, D. (2008). Contabilidad Administrativa. McGraw Hill. Décima edición. México. D.F.

Savall, H. (2013). Aspectos Generales del Modelo de Gestión Socioeconómica de Organizaciones. XIII Congresso Internacional de Costos - Porto - Portugal. 
Savall, H. \& Zardet, V. (2011). Por un trabajo más humano y eficiente. Modelo de gestión socioeconómica, 1-68. México: Instituto de Socio-Economía de las Empresas $y$ de las Organizaciones, ISEOR. Consultado el 18 de julio de 2017 enhttp:// editor.pbsiar.com/upload/PDF/por_un_trabajo_mas_humano_y_eficiente.pdf

Savall, H. (2006). Aspectos generales del modelo de gestión socioeconómica de organizaciones, 1-33. México: Laboratorio de Análisis Institucional del Sistema Universitario Mexicano, Laisum. Consultado el 26 de julio de 2017 en: http:// www.laisumedu.org/ DESIN_Ibarra/desin/pdf-seminario2006/ seminario2006-06a.pdf

Zardet, V. \& Krief, N. (2006). La teoría de los costos-desempeños ocultos en el modelo socioeconómico de las organizaciones, 1-20. México. Consultado el 21 de junio de 2017 de www.scielo.org.co/pdf/cuco/v15nspe39/v15nspe39a02.pdf 